\title{
A rigorous model of reflex function indicates that position and force feedback are flexibly tuned to position and force tasks
}

\author{
Winfred Mugge $\cdot$ David A. Abbink \\ Alfred C. Schouten · Julius P. A. Dewald • \\ Frans C. T. van der Helm
}

Received: 24 March 2009/ Accepted: 5 August 2009/Published online: 28 August 2009

(C) The Author(s) 2009. This article is published with open access at Springerlink.com

\begin{abstract}
This study aims to quantify the separate contributions of muscle force feedback, muscle spindle activity and co-contraction to the performance of voluntary tasks ("reduce the influence of perturbations on maintained force or position"). Most human motion control studies either isolate only one contributor, or assume that relevant reflexive feedback pathways during voluntary disturbance
\end{abstract}

W. Mugge and D. A. Abbink contributed equally to this work. A. C. Schouten and F. C. T. van der Helm supervised the research and commented on the manuscript. J. P .A. Dewald advised on the neurophysiologic background and implications.

W. Mugge $(\bowtie) \cdot$ D. A. Abbink · A. C. Schouten ·

F. C. T. van der Helm

Laboratory for Neuromuscular Control,

Department of Biomechanical Engineering,

Mechanical Engineering, Delft University of Technology,

Mekelweg 2, 2628 CD Delft, The Netherlands

e-mail: w.mugge@tudelft.nl

A. C. Schouten - F. C. T. van der Helm Laboratory of Biomechanical Engineering, Institute for Biomedical Technology (BMTI),

University of Twente, P.O. Box 217, 7500 AE Enschede,

The Netherlands

J. P. A. Dewald

Department of Physical Therapy and Human Movement

Sciences, Northwestern University, 645 N Michigan Avenue,

Suite 1100, Chicago, IL 60611, USA

J. P. A. Dewald

Department of Biomedical Engineering, Northwestern

University, 2145 Sheridan Rd, Evanston, IL 60208, USA

J. P. A. Dewald

Department of Physical Medicine and Rehabilitation,

Northwestern University, 345 E Superior St.,

Chicago, IL 60611, USA rejection tasks originate mainly from the muscle spindle. Human ankle-control experiments were performed, using three task instructions and three perturbation characteristics to evoke a wide range of responses to force perturbations. During position tasks, subjects $(n=10)$ resisted the perturbations, becoming more stiff than when being relaxed (i.e., the relax task). During force tasks, subjects were instructed to minimize force changes and actively gave way to imposed forces, thus becoming more compliant than during relax tasks. Subsequently, linear physiological models were fitted to the experimental data. Inhibitory, as well as excitatory force feedback, was needed to account for the full range of measured experimental behaviors. In conclusion, force feedback plays an important role in the studied motion control tasks (excitatory during position tasks and inhibitory during force tasks), implying that spindle-mediated feedback is not the only significant adaptive system that contributes to the maintenance of posture or force.

Keywords Human motion control .

Neuromusculoskeletal modeling - Afferent feedback .

Golgi tendon organs · Muscle spindles .

Muscle force feedback

\section{Introduction}

The presently accepted view is that Ia afferents act as excitatory input and $\mathrm{Ib}$ afferents as inhibitory input to the alpha motoneuron (Donelan and Pearson 2004). Is this the only possible role of Ia and Ib reflex pathways, or does this viewpoint result from the experimental conditions during which these pathways were studied (passive or statically loaded)? In experimental studies on walking 
patterns in decerebrate cats, evidence for a reversal from $\mathrm{Ib}$ inhibition to $\mathrm{Ib}$ excitation was found (Pearson and Collins 1993). Also from a theoretical study (Prochazka et al. 1997), it was concluded that force feedback is generally inhibitory (during stance), but that locomotion requires excitatory force feedback, augmenting the muscle stiffness. More recently, indirect evidence of adaptation in force feedback was found during human locomotion (Geyer et al. 2003; Grey et al. 2007), as well as experimental evidence suggesting that in humans $\mathrm{Ib}$ inhibition is only present in non-loaded situations such as during sitting and being in supine positions (Faist et al. 2006). However, in the latter case, possible contributions of Ia reflex pathways to the measured activity could not be conclusively ruled out.

The aim of this study was to simultaneously quantify contributions of both Ia and Ib pathway activity to the overall motion control behavior, not only during passive conditions, but also during tasks that require the preservation of posture or force. When attempting to understand the control of human motion during various functional voluntary tasks, one needs to investigate how the neuromuscular system acts as a whole and how the individual components of the neuromuscular system interact and contribute to its overall dynamic behavior. The field of control engineering provides tools to investigate dynamic systems, such as the neuromuscular system, and to successfully estimate the contribution of the individual system components (e.g., Kearney et al. 1997; Kiemel et al. 2006; Van der Helm et al. 2002; Van der Kooij et al. 2005).

Human motion control behavior in control theoretic terms

The essential components of the human motion control system can be compared to those of a robot: a linkage system (skeleton), actuators (muscles), a sensor system (proprioceptors) and a controller (the central nervous system or CNS), which is connected to the actuators and sensors (via the nerves). Several control strategies can be recognized, for example feed-forward (voluntary, planned and anticipated movements) and feedback (reflexive movements). Unpredictable disturbances, which occur during many activities of daily living, cannot be anticipated. This is why understanding mechanisms underlying sensory feedback is crucial (Shadmehr and Krakauer 2008).

Apart from relatively slow feedback that requires cortical processes (e.g., visual feedback), two principal strategies that allow humans to change the resistance of a limb to unpredictable perturbations exist:
1. instantaneous resistance, arising from passive structures and from (co-)contracted muscles (increased contraction enlarges the muscle visco-elasticity);

2. reflexive feedback from sensory organs in the muscle (proprioceptors) and from tactile sensors in the skin.

Both mechanisms are effective during posture maintenance, each with its advantages and disadvantages. Reflexive feedback only provokes muscle activity in response to a perturbation, making it energy-efficient. On the other hand, the inherent neural transport latency limits its effectiveness at higher frequencies. The effect of cocontraction is instantaneous and, therefore, also effective at higher frequencies at the expense of greater energy consumption.

The role of proprioceptive reflexes in motion control has been the subject of both physiological and experimental studies. Physiological studies have described the two known proprioceptors in detail: muscle spindles (MS) and Golgi tendon organs (GTO). MS are sensors parallel to the muscle fibers, which provide feedback to the CNS through Ia afferents (providing muscle stretch and velocity information) and through II afferents (mainly providing muscle stretch information). GTO are located in the muscle tendon and provide feedback through $\mathrm{Ib}$ afferents (providing muscle force information).

Many experiments have been performed that aim to isolate the reflexive contribution by studying electromyographical (EMG) and mechanical responses to perturbations. Literature shows that these responses depend on task instruction (Hammond 1956; Crago et al. 1976; Rothwell et al. 1980; Jaeger et al. 1982; Doemges and Rack 1992a, b; Abbink 2006; Pruszynski et al. 2008), the level of muscle contraction (Jaeger et al. 1982), the displacement amplitude (Cathers et al. 1999; Stein and Kearney 1995), the bandwidth of the perturbation signal (Van der Helm et al. 2002) and the mechanical load which the subject interacts with (De Vlugt et al. 2002). Comparisons and results between the many studies are difficult to interpret due to the abundance of experimental conditions: the measured joint, the type of perturbation (force or position, transient or continuous), and, often not explicitly stated, the task instruction. The reflexive contribution is usually attributed to MS-mediated feedback. Variations in the reflexive feedback contribution are ascribed to presynaptic Ia inhibition and gamma activation, according to the 'fusimotor set' hypothesis (Prochazka et al. 1985). Both are established physiological mechanisms for the muscle spindles. Physiological literature has shown mechanisms that may realize variations in GTO activity: "Ib fibers bear adequate synaptic equipment to receive substantial presynaptic inhibition" (Rudomin and Schmidt 1999). 
Evoking a wide range of motion control behavior

This study aims to evoke a broad range of control strategies of the subjects and as such provoke reflex modulation. For example, in dynamic experiments a position task can be expected to involve mainly position feedback, while a force task is likely to benefit from force feedback (Mugge et al. 2009). In this study, subjects were required to perform three tasks in face of continuous torque perturbations, according to the following task instructions:

- Force task (FT), i.e., maintain force, minimize force deviations.

- Relax task (RT), i.e., passive behavior, minimize muscle activity.

- Position task (PT), i.e., maintain position, minimize position deviations.

The dynamic control behavior as a whole can be quantified by the admittance: a measure of the displacement magnitude due to a force or torque, as a function of frequency. A small admittance (simply put: high stiffness) results in relatively small displacements in response to an external torque perturbation. This behavior is effective during PTs, such as posture maintenance or perturbation suppression. Daily life examples include steering a car on a bumpy road or holding an umbrella in a storm. It is hypothesized that during PT, the admittance is decreased as a result of co-contraction combined with excitatory MS feedback (Van der Helm et al. 2002). No information is yet available about the role of GTO feedback during PTs. RTs are hypothesized to result in negligible co-contraction and little MS and GTO feedback, effectively evoking a large admittance. FTs require a constant force to be exerted on an object (e.g., maintaining the pressure of a pencil on paper on the bus or holding a full cup of coffee during turbulence in a plane). During FT, force deviations are minimized, which is best accomplished by being compliant. It is hypothesized that FT performance is enhanced by active reflexes, helping to give way to the force perturbations, thereby reducing the overall stiffness of the system. Therefore, behavior with a large admittance is expected and even more compliant than during RT. Excitatory MS feedback and co-contraction increase stiffness (and damping) and are therefore counterproductive during FTs, while inhibitory GTO feedback decreases stiffness, improving FT performance. Therefore, it is hypothesized that FT entails inhibitory GTO feedback and little or no MS feedback, thus resulting in assistive muscle contractions, reducing the contact force in the absence of co-contraction. The hypotheses are summarized in Table 1.

To test these hypotheses, first the admittances of ten subjects performing the three tasks in face of continuous random torque perturbations were experimentally obtained.
Table 1 Hypothesized effects of task instruction

\begin{tabular}{llll}
\hline Task & Position & Relax & Force \\
\hline Admittance & Small & Large & Very large \\
Co-contraction & High & Low & Low \\
MS feedback & Excitatory & Absent & Absent \\
GTO feedback & Absent & Absent & Inhibitory \\
\hline
\end{tabular}

Perturbations with various frequency spectra were used, since previous research has shown that the admittance during PTs strongly depends on perturbation bandwidth (Van der Helm et al. 2002). To parameterize the underlying physiology, a neuromusculoskeletal model was fitted to the admittances. The validated model yielded quantitative parameters for the velocity and position feedback gains (corresponding to Ia-activity) and force feedback gains (corresponding to Ib-activity).

\section{Methods}

Experiment

\section{Subjects}

Ten subjects (five male and five female) with an age range of 20-28 years, without medical record of neurological disease or ankle injuries, participated in the experiment. Subjects gave informed consent and received financial compensation.

\section{Experimental setup}

Subjects were positioned comfortably in a car-driving posture (Fig. 1), such that their right leg was aligned with the gas pedal and the pedal depression was $10^{\circ}\left(\approx 90^{\circ}\right.$ ankle flexion angle). The foot was firmly secured to the gas pedal. The gas pedal system was based on a commercially available force-controlled actuator, by which a virtual mass-spring-damper system can be simulated and torque perturbations applied (Moog-FCS, The Netherlands). The gas pedal served as a moment arm, translating the torque perturbations to force perturbations at the foot. In the cardriving posture, the movements of the pedal caused small ankle rotations $\left(\mathrm{SD} \sim 1.0^{\circ}\right.$ ), accompanied by negligible (vertical) movements of the knee. The small movements ensured quasi-linear behavior, enabling linear analysis. This study was performed using a gas pedal stiffness of $17.5 \mathrm{Nm} / \mathrm{rad}\left(\approx 1.6 \mathrm{~N} /{ }^{\circ}\right.$ force at foot contact $)$. The pedal damping $\left(7.5 \times 10^{-4} \mathrm{Nms} / \mathrm{rad}\right)$ and virtual inertia $\left(5.1 \times 10^{-3} \mathrm{~kg} \mathrm{~m}^{2}\right)$ were kept small. A bias force $(12 \mathrm{~N})$, together with the pedal stiffness, compensated for the 
Fig. 1 Experimental setup. The upper panel shows a seated subject in front of the monitor with task-related information. The lower left panel shows a close-up of the lower limb, with the foot strapped to the pedal. The lower right panel shows a schematic representation of the setup, with $d_{1}$ the distance between force sensor and rotation point $\left(P_{r}\right), d_{2}$ the distance between the contact point on the gas pedal $\left(P_{c}\right)$ and the pedal rotation point $\left(P_{r}\right)$ (estimated at $188 \mathrm{~mm}$ ). The measured signals (perturbation torque $D$, contact torque $T_{c}$ and pedal rotation $\left.\theta_{\text {pedal }}\right)$ are discussed in the text
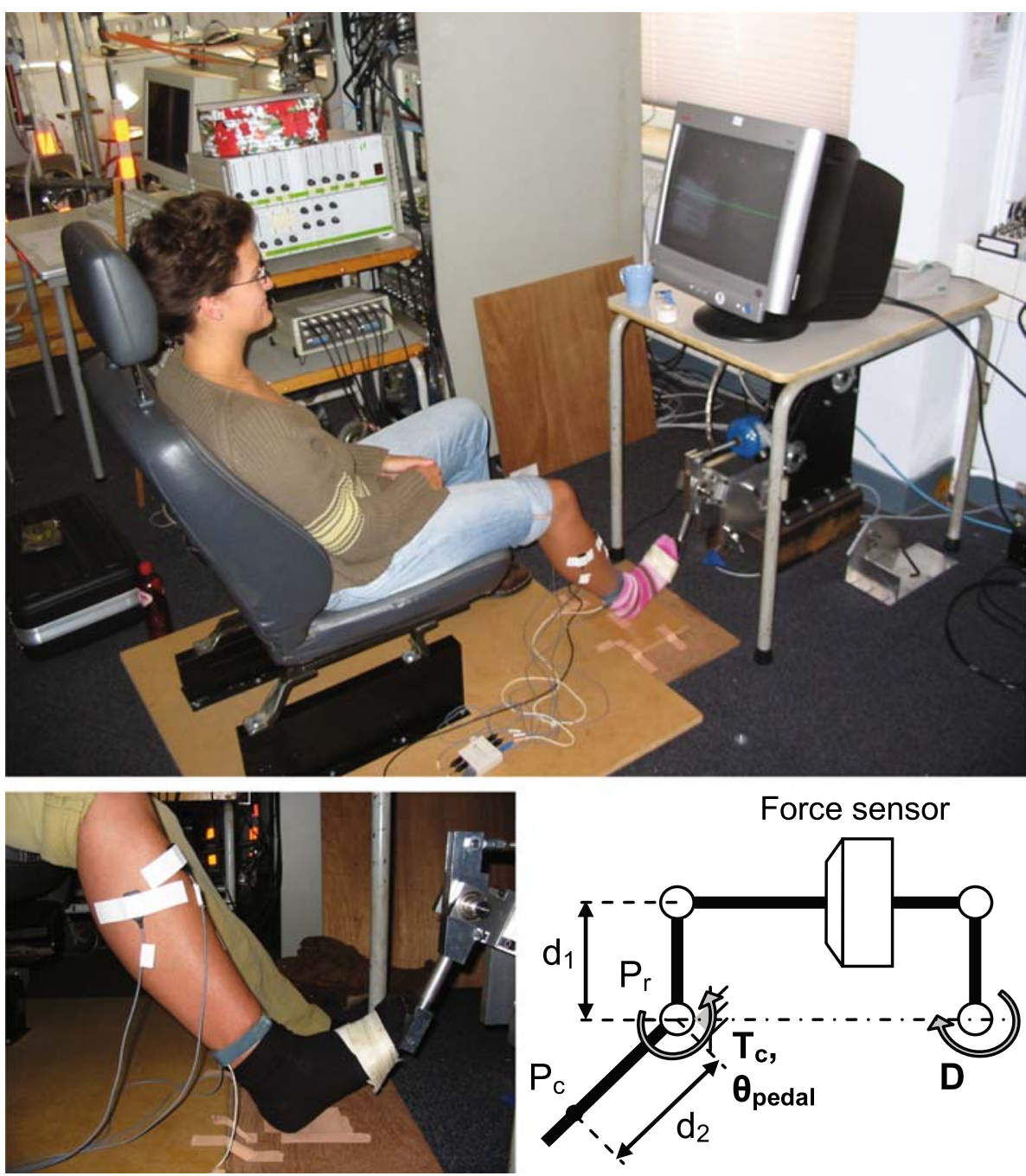

weight of the foot. This allowed the subject to relax the lower leg muscles in the desired configuration.

Before the experiment, the subjects received specific instructions on how to perform the three tasks: the force task (FT), relax task (RT) and the position task (PT). To prevent drift from the reference, visual feedback was presented on a 17-inch monitor mounted in front of the subject during FT and PT. During PT, the gas pedal angle was plotted against a horizontal reference line indicating the target angle $\left(10^{\circ}\right.$ pedal depression), whereas during FT the torque exerted on the gas pedal was plotted against a horizontal reference line indicating the target torque. The target torque was equal to the weight of the foot at the desired position (approximately $28 \mathrm{~N}$ at foot contact, again $10^{\circ}$ pedal depression), so that no muscle force was required. During RT, the monitor was turned off.

For each of the three tasks, three torque perturbation signals were designed yielding nine conditions (each a combination of a perturbation bandwidth and a task instruction). Each condition was presented to the subject four times for averaging purposes; yielding 12 trials per task (and 36 trials in total). The order in which subjects had to perform the three tasks was randomized for each subject, the order of the trials was randomized as well, but grouped to corresponding task instructions to improve overall task perception. Every task instruction was accompanied by a 5 -min break to prevent fatigue and $10 \mathrm{~min}$ of training.

\section{Signal recording}

The torque on the gas pedal $T_{c}(t)$ was measured by a force sensor (Interface, 1210BXH-300, positioned at a moment arm $\left(d_{1}\right)$ of $\left.76.2 \mathrm{~mm}\right)$. The gas pedal angle $\theta_{\text {pedal }}$ was measured by an angle encoder. The signals were recorded via analog-to-digital conversion at $250-\mathrm{Hz}$ sample frequency and digitally stored. Furthermore, differential surface electrodes (Delsys) recorded EMG of four muscles: ankle plantarflexors (pushing down), gastrocnemius medialis (GM), gastrocnemius lateralis (GL) and soleus (SOL); and dorsiflexor (pulling up), tibialis anterior (TA). The 
EMG signals were pre-amplified, low-pass filtered at $450 \mathrm{~Hz}$ and high-pass filtered at $20 \mathrm{~Hz}$, before being digitized at $1,250 \mathrm{~Hz}$ by a separate system. For each trial, the recorded EMG was rectified, normalized to the EMG during maximum voluntary contraction (MVC) and finally averaged over time. Dorsiflexor EMG consisted of EMG of TA only, while plantarflexor EMG was the average of EMG of GM, GL and SOL muscles. The EMG during MVC was obtained before and after the experiment and averaged. The EMG normalization allowed for inter-subject comparison.

The first $3 \mathrm{~s}$ and the last second of all measured signals were excluded from analysis to reduce the influence of possible transient effects (due to the onset of the perturbation or an imminent stop).

\section{Perturbation signals}

The three perturbation signals were designed in the frequency domain to have rectangular spectra containing dominant power from 0.1 to $0.7,1.2$ and $2.0 \mathrm{~Hz}$. Power was applied to two adjacent frequency points, to enable frequency averaging to reduce estimator variance (Jenkins and Watts 1968; Pintelon and Schoukens 2001). Inverse Fourier transform yielded unpredictable time signals with 37.0 s duration.

All perturbations were supplemented with a reduced level of power up to $40 \mathrm{~Hz}$, according to the Reduced Power Method (Mugge et al. 2007) enabling system identification and parameter fits at higher frequencies, while still evoking behavior adapted to low-frequent perturbations.

To prevent amplitude non-linearities, the standard deviation of pedal depression was equalized for each condition by scaling of the torque perturbation magnitude (the correct scaling was determined during training trials). The reduced power level was kept at a constant absolute level to ensure a good signal-to-noise ratio. As a result, reduced power ranged from $20 \%$ of the dominant power during FT to $2.0 \%$ during PT.

\section{Non-parametric system identification}

The admittance was estimated in the frequency domain, using a closed-loop frequency domain identification procedure (Van der Helm et al. 2002), according to:

$\hat{H}_{T \theta}(f)=\frac{\hat{S}_{D \theta}(f)}{\hat{S}_{D T_{c}}(f)}$,

in which $\hat{S_{D \theta}}(f)$ denotes the cross-spectral density of external torque perturbation $D(f)$ and gas pedal angle $\theta_{\text {pedal }}(f)$, and $\hat{S_{D T}}$ denotes the cross-spectral density of external torque perturbation $D(f)$ and gas pedal torque $T_{c}(f)$.
The procedure estimates the admittance without any predefined model structure, but does assume linearity, which can be checked by the coherence according to:

$\hat{\Gamma}_{D \theta}^{2}(f)=\frac{\left|\hat{S}_{D \theta}(f)\right|^{2}}{\hat{S}_{D D}(f) \cdot \hat{S}_{\theta \theta}(f)}$

The squared coherence function $\hat{\Gamma}_{D \theta}^{2}$ ranges from zero for systems with no linear relation to one for a linear system without noise (linearization or measurement noise).

\section{Model parameterization}

To obtain physiologically relevant parameters, a neuromusculoskeletal model was fitted to the experimental data. The model describes the admittance with 13 physiologically interpretable parameters: 5 that represent condition-dependent parameters and 8 that represent condition-independent parameters. The condition-dependent parameters describe the strength of reflexive feedback from muscle spindles (position feedback gain $k_{p}$, velocity feedback gain $k_{v}$ ), Golgi tendon organs (force feedback gain $k_{f}$ ) and muscle viscoelasticity (muscle stiffness $k_{a}$, muscle viscosity $b_{a}$ ). The condition-independent parameters all described processes that were assumed not to change with task instruction or perturbation bandwidth, but should be able to vary between subjects: i.e., ankle inertia $\left(I_{a}\right)$, tendon stiffness $\left(k_{\text {tendon }}\right)$, reflexive time delays (for MS $\tau_{\mathrm{ms}}$, for GTO $\tau_{\mathrm{gto}}$ ), secondorder activation dynamics (relative damping $d_{a}$, eigen-frequency $f_{a}$ ) and contact dynamics (damping $b_{c}$ and stiffness $k_{c}$ ). The full model has been described previously (Schouten et al. 2008) and is shown in Fig. 2. The model linearization and derivation are described in Appendices 1 and 2. The outcome of a parameter fit can strongly depend on the choice of model structure and parameter boundaries. As a result, an incorrect choice could affect the validity of the conclusions of this study. A substantial part of "Discussion" will address the ability of the model to describe the measured behavior, as well as to what extent the conclusions depend on the chosen model assumptions. Particularly, the effect of long-latency reflexes instead of short-latency reflexes and the omission of GTO feedback are assessed.

The complete neuromusculoskeletal model describes the admittance according to:

$$
\begin{aligned}
H_{\text {model }} & =\frac{\theta_{\text {pedal }}}{T_{\mathrm{c}}} \\
& =\left[H_{\mathrm{c}}^{-1}+\frac{1}{H_{\mathrm{i}}+\left[H_{\mathrm{ve}}+H_{\mathrm{ms}} H_{\mathrm{act}}\right] H_{\text {filter_tendon }} H_{\text {filter }}}\right]
\end{aligned}
$$

with $H_{\mathrm{c}}$ describing the contact dynamics, $H_{\mathrm{i}}$ the inertial dynamics, $H_{\mathrm{ve}}$ the muscle visco-elasticity, $H_{\mathrm{ms}}$ the muscle 


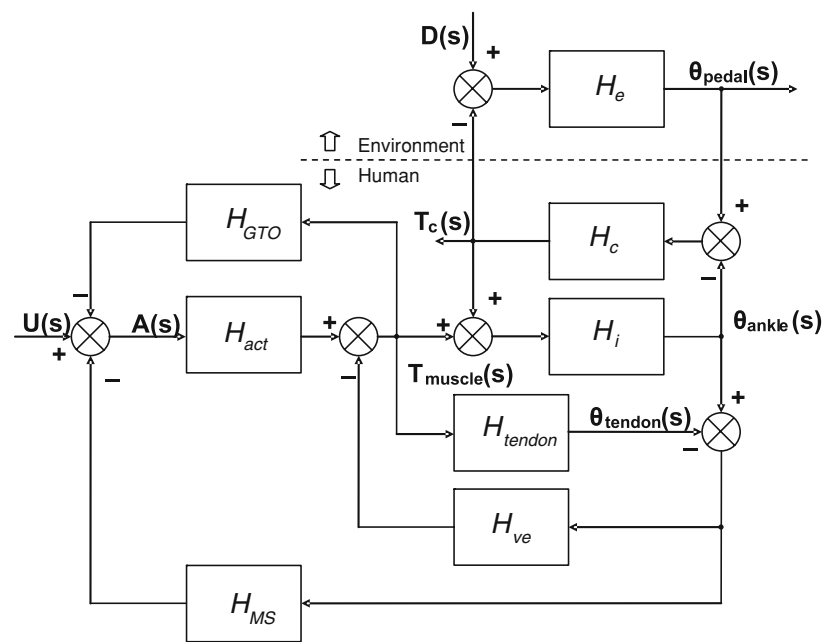

Fig. 2 Model of neuromusculoskeletal system in interaction with a gas pedal (with dynamics $H_{e}$ ). The linear block scheme details the contributions to human admittance in the frequency domain, where $\mathrm{s}$ is the Laplace operator. All parameters and signals are discussed in the text ("Model parameterization"), and an in-depth derivation can be found in Appendix 1

spindle activity and $H_{\text {act }}$ the muscle activation dynamics. The equation was condensed using the following equations:

$$
\begin{aligned}
H_{\text {filter_tendon }} & =\frac{H_{\text {tendon }}}{H_{\text {tendon }}+H_{\mathrm{ve}}} \\
H_{\text {filter }} & =\frac{1}{\left[1-H_{\text {filter_tendon }}\left[-H_{\text {gto }}-\left(\frac{H_{\mathrm{ms}}}{H_{\text {tendon }}}\right)\right] H_{\text {act }}\right]}
\end{aligned}
$$

where $H_{\text {tendon }}$ describes the tendon stiffness, and $H_{\text {gto }}$ the Golgi tendon organ activity.

\section{Parameter fit procedure}

The parameter fit was done in the frequency domain, to the admittances estimated from noise-free signals generated using auto-regressive models (Van den Hof et al. 1995). The 13 parameters were identified by fitting the model on the frequency response function (FRF) of an ARMAX approximation of the measured signals, a technique known to improve the fit (De Vlugt et al. 2006).

The fit procedure was guided by parameter boundaries (see Table 2), which served to prevent unrealistic parameter values and bad convergence. As a fit criterion, the total of the squared logarithmic difference in admittance was minimized (Pintelon et al. 1994) over all conditions and frequencies, fitting all parameters for each subject in one optimization, according to:

$E=\sum_{\operatorname{cond}(1)}^{\operatorname{cond}(k)} \sum_{\operatorname{freq}(1)}^{\operatorname{freq}(m)}\left|\log \left[\frac{H_{\text {ARMAX }}}{H_{\text {Model }}}\right]\right|^{2}$ with freq $(l)$ representing the lowest frequency, freq $(m)$ the highest, cond( $(1)$ the first condition (task $\times$ bandwidth), $\operatorname{cond}(k)$ the last condition, $H_{\text {ARMAX }}$ the FRF of the ARMAX approximation to the measurement data and $H_{\text {model }}$ the FRF of the model. The error $E$ was summed over all frequencies and all experimental conditions. Five parameters were estimated for each of the nine experimental conditions and 8 parameters were kept constant over all conditions, resulting in a total of 53 parameters per subject.

\section{Parameter fit validation}

As a measure of the validity of the ARMAX approximation as well as of the model fit, the variance accounted for (VAF) was calculated for both steps (Eq. 5). The VAF relates how much of the variance of a signal $\left(u_{1}\right)$ is captured by a predicted signal $\left(u_{2}\right)$. A VAF of $100 \%$ means that $u_{2}$ perfectly describes $u_{1}$.

The VAF for the position was calculated from measured position $\left(u_{1}\right)$ and the model-predicted position $\left(u_{2}\right)$, yielded by time domain simulation of the model with the fitted parameters, according to:

$\mathrm{VAF}=\left[1-\frac{\sum_{q(1)}^{q(n)}\left(u_{1}(t)-u_{2}(t)\right)^{2}}{\sum_{q(1)}^{q(n)}\left(u_{1}(t)\right)^{2}}\right] \cdot 100 \%$

with $q(1)$ representing the first sample and $q(n)$ the last sample of the time signal. In this study, the first sample was chosen after $1.6 \mathrm{~s}$ to exclude transient effects of filters (ARMAX) and integrators (parametric model). The same procedure was followed to calculate the VAF for force.

\section{Statistical analysis}

The effect of task and perturbation bandwidth on the admittance was tested for significance using a repeated measures ANOVA $(p<0.05)$ on the magnitude of the admittance over all subjects, for each frequency point. Effects on the five condition-dependent parameters were also tested for significance using a repeated-measures ANOVA over all subjects, for each parameter.

\section{Results}

Time domain analysis

Figure 3 shows a time segment of a few seconds, to illustrate the difference between FTs and PTs. First of all, it shows the task-dependent difference in magnitude of the torque perturbations and contact torques at the same 
Fig. 3 Time domain results for a typical subject during FT (left) and PT (right). The top panels show the perturbation torque $D$ (solid line) and contact torque $T_{c}$ (dashed line) at the pedal rotation point. The middle panels show the EMG activity of plantarflexors (averaged) and the dorsiflexor, scaled to a percentage of maximal EMG activity. The bottom panels show the pedal rotation (dorsiflexion is defined as positive). For this plot, all signals are filtered with a thirdorder Butterworth filter at $3 \mathrm{~Hz}$
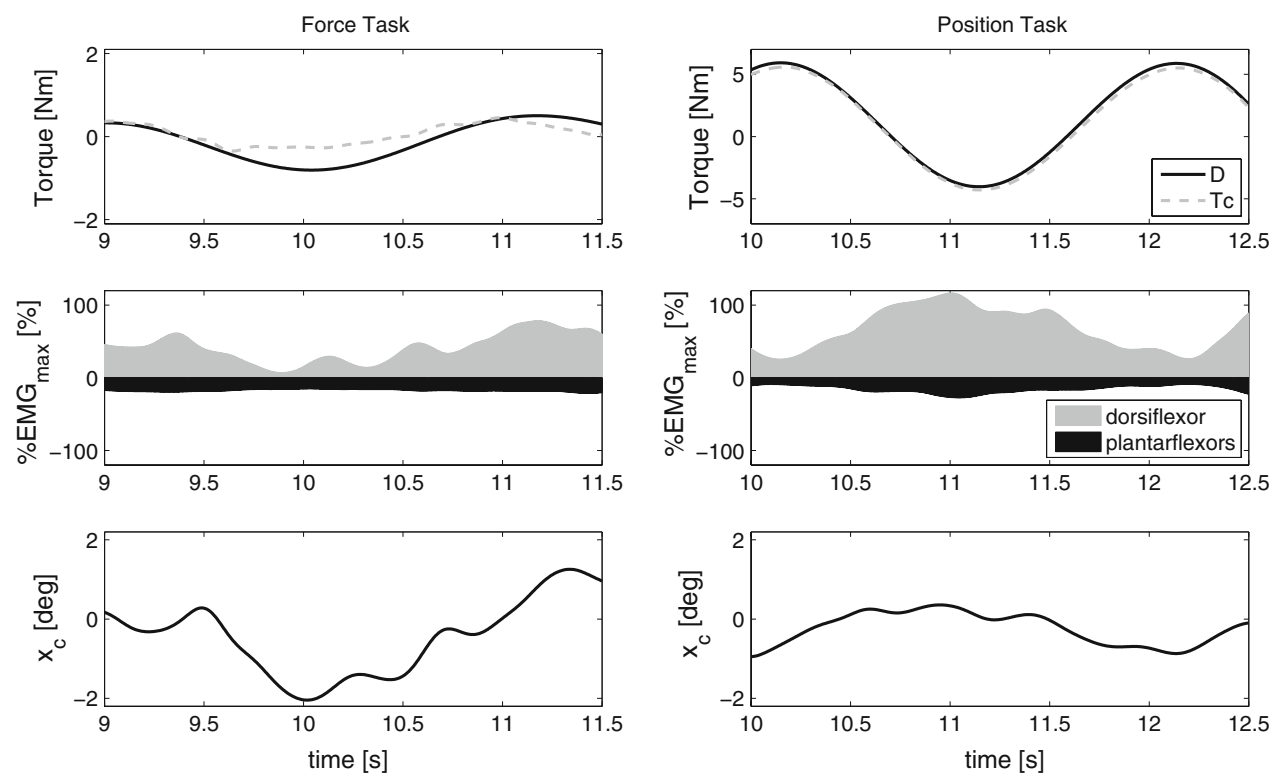

angular deviations: an indication of a difference in admittance. Second, during FTs, the resultant torque $T_{c}$ is almost zero, while during PTs $T_{c}$ counteracts the perturbation more. Also, PTs entail much EMG activity.

An intriguing difference between FTs and PTs, is that during FTs the EMG activity is in phase with the torque, while during PTs it is in anti-phase. In other words, during FTs subjects actively used muscle activation strategies that give way to the perturbations, while during PTs subjects counteracted the perturbations.

Figure 4 shows the mean EMG level for all conditions. During PTs significantly $(p<0.01)$ more EMG activity was found compared to FTs and RTs, indicating increased muscle co-contraction. FTs entail a slight, but significant $(p<0.01)$, increase in EMG with respect to RTs. Within the tasks no significant effect of bandwidth on the mean EMG level was found.

Non-parametric frequency domain identification

As hypothesized, the admittance was substantially influenced by task instruction. Figure 5 presents the admittance during four repetitions of one subject performing the three task instructions. The largest admittance occurs during FT (i.e., minimized force deviations), a smaller, but still large, admittance during RT (i.e., minimized muscle activity), and a small admittance during PT (i.e., minimized position deviations). These task-dependent changes in admittance were consistent over all subjects: a significantly $(p<0.01)$ higher admittance was found for FTs with respect to RTs up to $3.0 \mathrm{~Hz}$, and a significantly $(p<0.01)$ lower admittance was found for PTs with respect to RTs up to $1.0 \mathrm{~Hz}$. The (squared) coherence was high (not shown, $>0.9$ for all frequencies) indicating that the input-output behavior can be considered linear with low levels of noise.

In addition, significant effects of perturbation bandwidth were found. As can be seen from Fig. 6, perturbations with dominant power up to a lower frequency result in improved task performance: an increased admittance during FTs and a decreased admittance during PTs.

Parametric frequency domain identification

The eight condition-independent parameters (limb inertia, tendon stiffness, reflexive time delays, muscle activation dynamics and contact visco-elasticity) are shown in
Fig. 4 Results of the EMG measurements normalized to MVC. Mean and standard deviations over all subjects are shown. The panels present FT, $\mathrm{RT}$ and PT from left to right. Inside each window from left to right, the three bandwidths, 0.7 , 1.2 and $2.0 \mathrm{~Hz}$ are presented

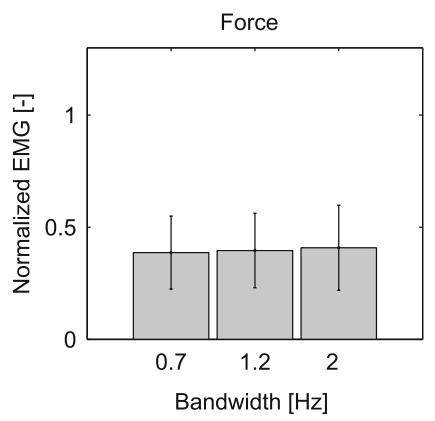

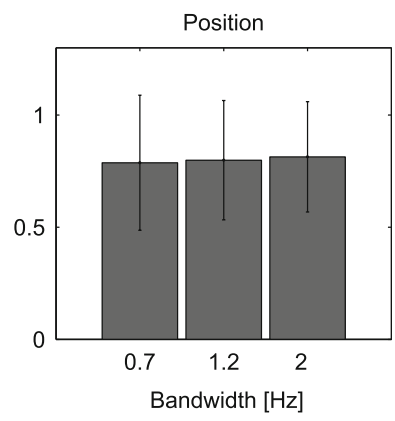


Fig. 5 Task effect on admittance of a typical subject. For each of the four repetitions, the magnitude (top panel) and phase (bottom panel) are shown for FT (dashed line), RT (solid line) and PT (dash-dotted line). The shown admittance is the response to a perturbation with a bandwidth of $0.7 \mathrm{~Hz}$ (supplemented with reduced power up to $40 \mathrm{~Hz}$ )
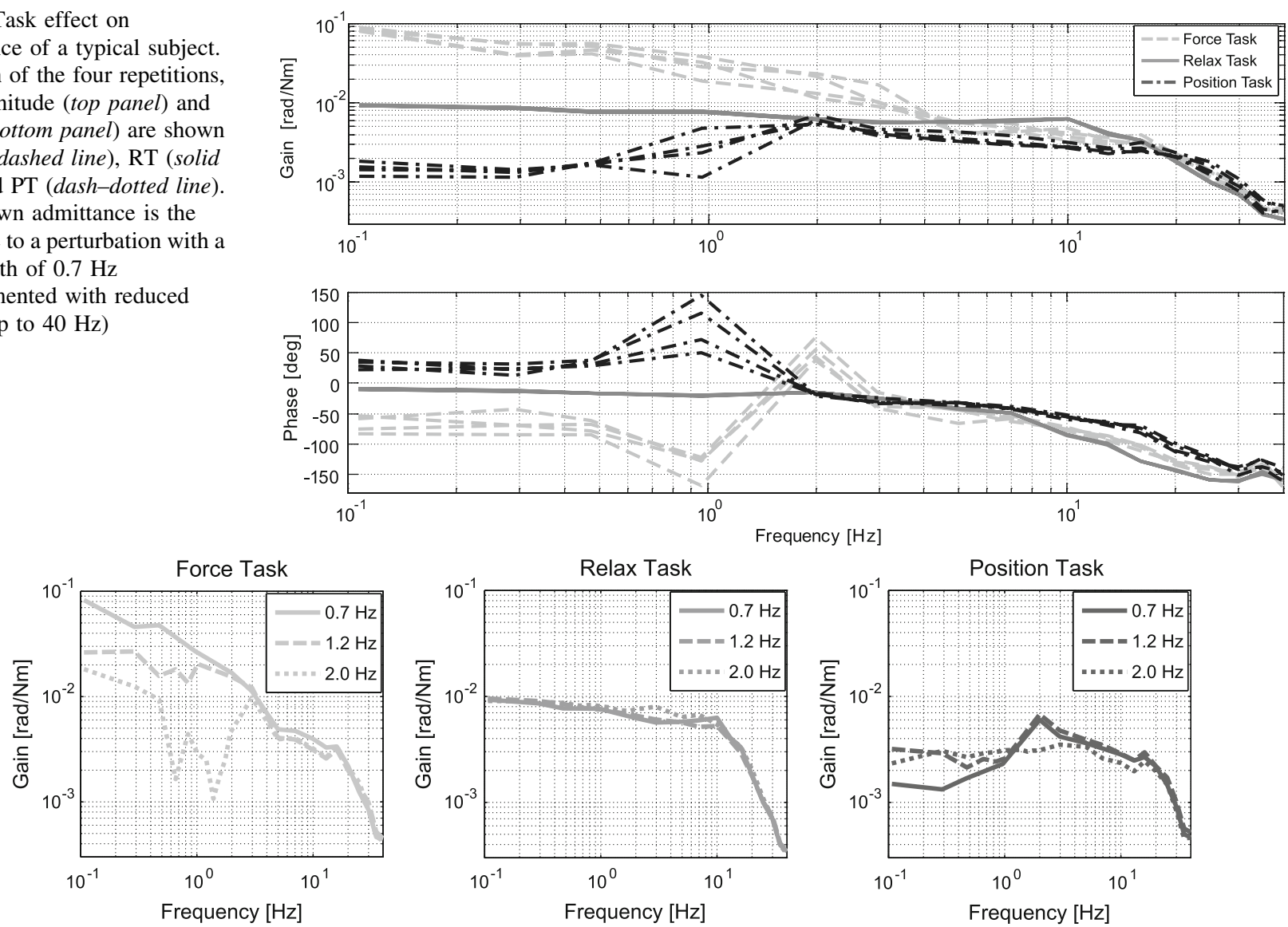

power up to $0.7,1.2$ and $2.0 \mathrm{~Hz}$ ). The shown admittance is the average over the four repetitions subject. The magnitude of the admittance is shown for FT (left), RT (middle) and PT (right) at all perturbation bandwidths (with dominant

Table 3 (mean \pm SD over subjects). Figure 7 shows the five condition-dependent parameters (position and velocity feedback originating from MS, force feedback originating from GTO, and muscle visco-elasticity). The effect of task instruction on the condition-dependent parameters is in accordance with the hypotheses (inhibitory GTO during FT, excitatory MS during PT), except for two remarkable results. In addition to the inhibitory GTO feedback during FT, inhibitory MS feedback was found, and in addition to the excitatory MS feedback during PT, excitatory GTO feedback was found.

\section{Adaptations in force feedback from GTO}

The effect of task instruction on the force feedback gain $k_{f}$ was substantial. The gain was even shown to change sign (i.e., from inhibitory to excitatory). Significantly $(p<0.05)$ more inhibiting GTO feedback $\left(k_{f}=1.2\right)$ was found during FTs at bandwidths 0.7 and $1.2 \mathrm{~Hz}$ with respect to all other conditions, including the FT with $2.0 \mathrm{~Hz}$ perturbation bandwidth $\left(k_{f}=0.2\right)$, indicating a bandwidth effect during FTs.
Significantly $(p<0.01)$ more excitatory GTO feedback $\left(k_{f}=-1.0\right)$ was found during PTs with respect to RTs $\left(k_{f}=0.1\right)$ and FTs $\left(k_{f}=1.2\right)$. A significant $(p<0.05)$ bandwidth effect on the GTO gain was found during PTs as well.

\section{Adaptations in position and velocity feedback from MS}

The results for the MS position and velocity feedback gains ( $k_{p}$ and $k_{v}$ ) show more variability, and were generally less interpretable. The gains did not change significantly with bandwidth, and a task effect was only found for $k_{v}$ : during PTs an inhibitive gain $\left(k_{v}=-17 \mathrm{Nm} \mathrm{s} / \mathrm{rad}\right)$ was estimated for $0.7 \mathrm{~Hz}$ and $1.2 \mathrm{~Hz}$ perturbation bandwidth, significantly $(p<0.05)$ lower than for other conditions.

\section{Adaptations in muscle visco-elasticity}

Even though muscle viscosity and elasticity are physiologically related, they were modeled independently. Still, the parameters jointly changed with task instruction: during PTs, substantially increased stiffness $\left(k_{a}=550 \mathrm{Nm} / \mathrm{rad}\right)$ 

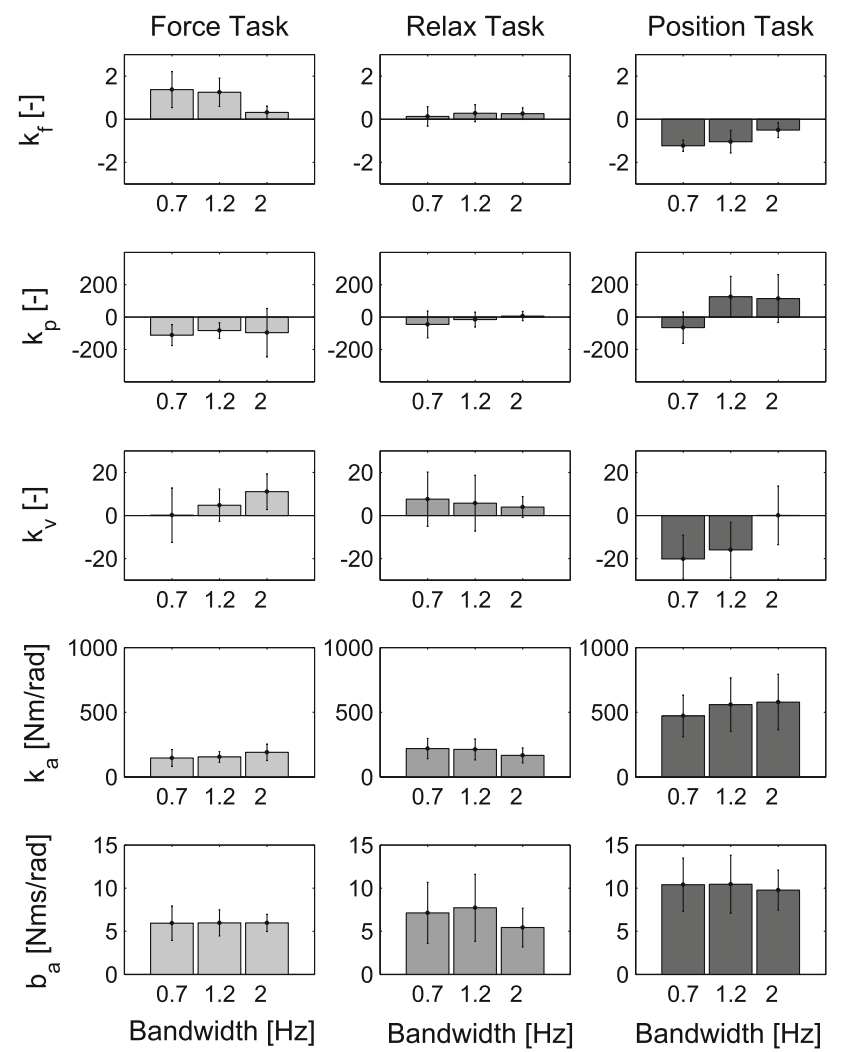

Fig. 7 Parametric identification results for the five condition-dependent parameters. Mean and standard deviation over all subjects are shown for each parameter. The panels present FT, RT and PT from left to right. Inside each panel, the fitted parameter for each of the three bandwidths $(0.7,1.2$ and $2.0 \mathrm{~Hz}$, from left to right $)$ is presented

and damping ( $b_{a}=10 \mathrm{Nm} \mathrm{s} / \mathrm{rad}$ ) were found compared to FTs and RTs $\left(k_{a}=180 \mathrm{Nm} / \mathrm{rad}, p<0.01\right.$ and $b_{a}=7$ $\mathrm{Nm} \mathrm{s} / \mathrm{rad}, p<0.05)$. Bandwidth did not significantly influence the muscle visco-elasticity. These results correspond with the EMG level results of the time domain analysis, and show that the parameter estimation retrieved the physiological relation between muscle viscosity and elasticity.

\section{Validation}

The fit procedure yielded a parameterized model that accurately captures the dynamics: Fig. 8 shows the magnitude and phase of the admittances during FT, RT and PT as estimated with the parameter fit (solid line), with the ARMAX-approximated signals (dashed line) and with the measured signals (dotted line).

The VAFs between the model-predicted signals (contact torque and angular position) and the ARMAX approximations are presented in Table 4, together with the VAFs between the ARMAX approximations and the measured signals. All VAFs were generally very good for RTs and PTs, but lower during FTs.

\section{Discussion}

The effect of task effect and perturbation bandwidth on admittance

Consistent with previous studies, task instruction had a substantial effect on joint admittance (Hammond 1956; Crago et al. 1976; Rothwell et al. 1980; Jaeger et al. 1982; Doemges and Rack 1992a, b; Abbink 2006; Pruszynski et al. 2008). As hypothesized, for all subjects the largest admittance was found during FTs, a smaller admittance during RTs, and the smallest admittance was found during PTs. During FTs, slightly more EMG activity was found compared to RTs (see Fig. 4), indicating that subjects used their muscles to actively give way. During PTs, much more EMG activity for all relevant muscles was found compared to RTs.

Similar results were found for FT, RT and PT in singlejoint experiments in the same (Abbink 2007) and other joints (wrist) and in multi-joint systems such as controlling a steering column and side-stick in an airplane (Lasschuit et al. 2008), in which also an increased admittance for the FT was found with respect to the RT.

The perturbation bandwidth significantly influenced the admittance for both FTs and PTs: the lower the perturbation bandwidth, the better the subjects performed their task. This bandwidth effect was previously reported for PTs during arm posture control (Van der Helm et al. 2002), where it was explained by a trade-off between performance and stability. Theoretically, a large amount of position feedback activity would be optimal during a PT. However, due to the neural conduction and synaptic delays, position (and velocity) feedback causes oscillations at its eigenfrequency, which is counterproductive to task performance. Therefore, when a perturbation contains substantial power near this eigen-frequency, the feedback gains are suppressed. The small bandwidth perturbations in this study evoke low-frequent admittance changes that are substantially larger than reported in previous literature (Colebatch and McCloskey 1987; Hore et al. 1990). It is presently unclear why these differences exist. Possibly, the present method elicited more reflexive activity due to the provoked functional behavior (task instruction with motivating visual feedback) and the perturbation properties that caused reflexive activity to be exceptionally effective (Mugge et al. 2007).

Unexpected result: excitatory GTO feedback

The model results section shows that the model that included inhibitory as well as excitatory MS and GTO yields accurate fits for all conditions. This may be surprising, given the prevailing neurophysiologic view of 

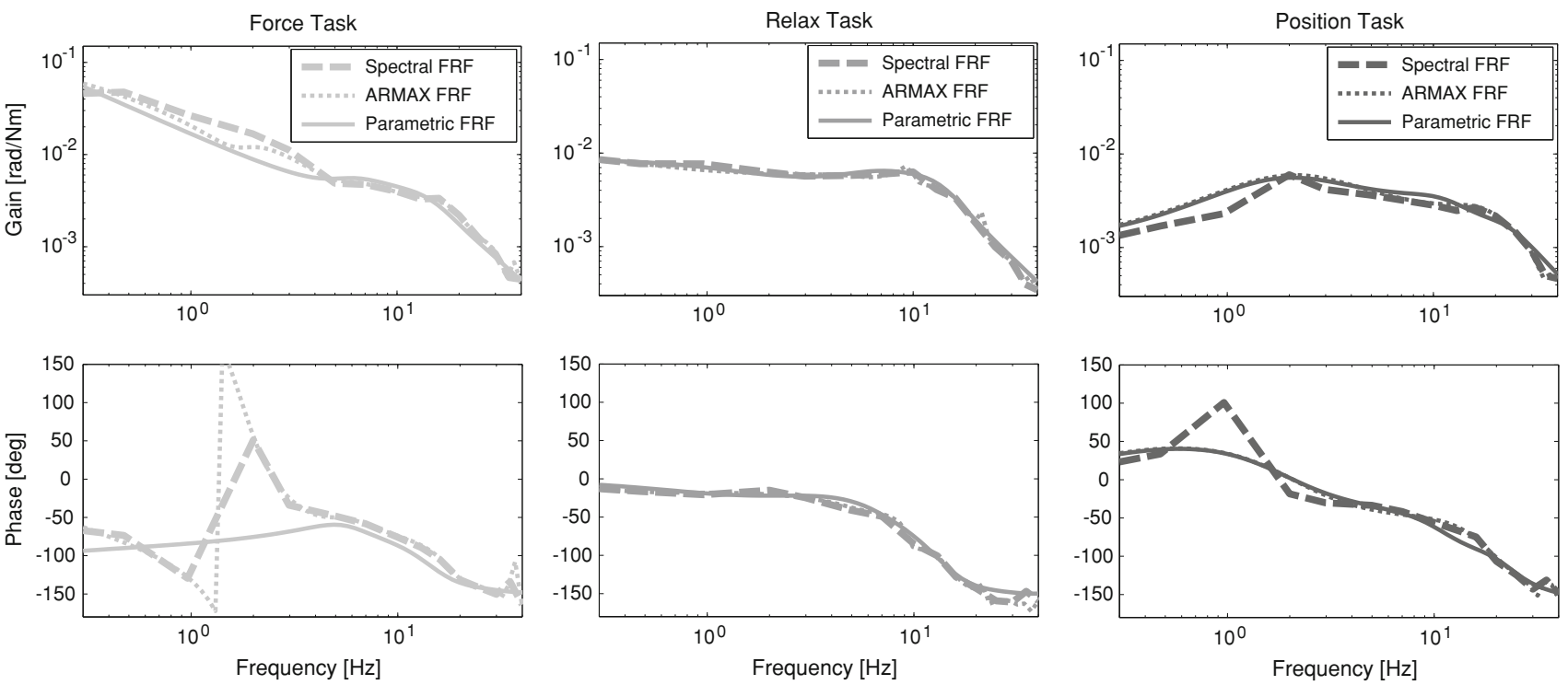

Fig. 8 Frequency domain validation of the parametric fit for a typical subject. Magnitude and phase of the parameterized model (solid line) are shown on top of the frequency response function from the ARMAX-approximated signals (averaged over four repetitions, represented by a dotted line). As a reference, the spectral frequency

GTO being essentially inhibitory and MS excitatory. Could a model with only MS yield the same fits? The solid line in the left panel of Fig. 9 shows the results of a typical parameter fit for PT, using a model comprising all parameters except the GTO force feedback gain $k_{f}$. The reduced model could not accurately capture the dynamics of the admittance during FT and PT, resulting in bad fits, substantially reduced VAFs and unrealistic parameter values. Could a model with only inhibitory GTO yield accurate fits? The implementation of strictly inhibitory GTO muscle force feedback did result in accurate fits for FT; however, the behavior during PT can only be accurately described with a model that incorporates excitatory muscle force feedback.

Neurophysiologic background for inhibitory and excitatory reflexive feedback

Is there a physiological basis for the assumption that MS activity and GTO activity can be both excitatory and inhibitory? The overall effect of Ia afferent information on the efferent output of the alpha motor neuron originates from a direct excitatory connection (monosynaptic, but affected by presynaptic inhibition) and from indirect connections through excitatory and inhibitory interneurons in the spinal cord (Jankowska and McCrea 1983). The pathways with inhibitory interneurons can result in an overall inhibitory effect of Ia on the alpha motor efferent output (Stienen et al. 2007). Shared interneuronal circuits resulting in inhibitory and excitatory effects of $\mathrm{Ib}$ afferent input response function estimated from the measured signals (averaged over four repetitions) is shown by the dashed line. The admittances in response to a $0.7 \mathrm{~Hz}$ perturbation bandwidth are presented for FT (left), RT (middle) and PT (right)
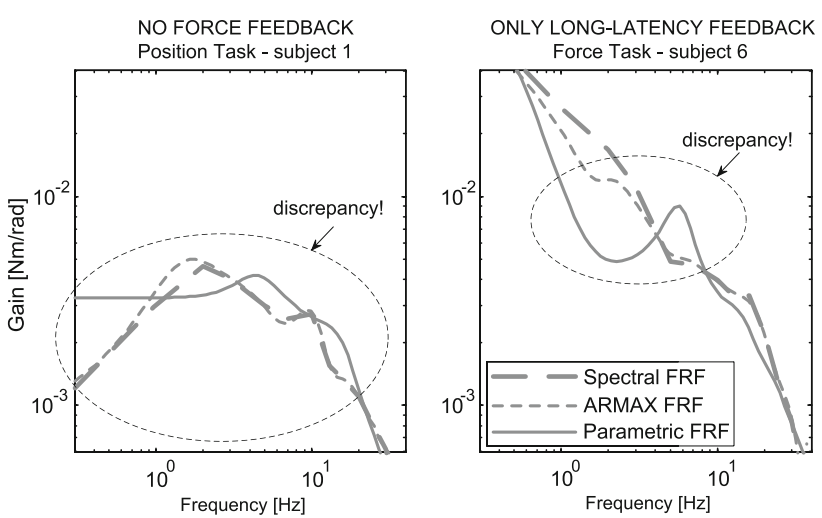

Fig. 9 Model fits using incorrect neuromusculoskeletal model structures. Magnitude and phase of the parameterized models (solid lines) are shown on top of the frequency response functions from the ARMAX-approximated signals (averaged over four repetitions, represented by dotted lines). As a reference, the spectral frequency response functions estimated from the measured signals (averaged over four repetitions) are shown by dashed lines. The behavior was measured in response to the $0.7 \mathrm{~Hz}$ perturbation bandwidth. Left: fit results of a model without GTO muscle force feedback on data of a typical subject performing a PT. Right: fit results of a model with long-latency instead of short-latency reflexes on data of a typical subject performing an FT

to the alpha motor neurons have been described (Jankowska and McCrea 1983) in the cat.

Experimental findings from literature report excitatory GTO feedback during walking (Faist et al. 2006; Grey et al. 2007). Apparently, the same neurophysiologic mechanisms responsible for excitatory Ib feedback during walking apply also for the non-walking PT in this study. 
More specifically, results from our parameter fit suggest an important behavioral role for GTO activity in face of low-frequent perturbations: inhibitory to increase the admittance during FTs and excitatory to decrease the admittance during PTs. The slightly excitatory MS position feedback during PTs did not differ significantly from the other tasks, and only the inhibitory velocity feedback was significant. This inhibitory feedback was not expected, since on its own it increases the admittance, degrading PT performance. A separate model sensitivity analysis at the ankle using the NMCLab Graphical User Interface (Schouten et al. 2008) yielded evidence that slightly inhibitory velocity feedback has a stabilizing effect near the GTO's eigen-frequency, allowing more excitatory GTO feedback: an interesting notion that demands additional research. Moreover, it also revealed that $k_{p}$ and $k_{v}$ had less impact on the fit accuracy than $k_{f}$ (which may explain the large variability in the estimated muscle spindle feedback gains).

The effect of task and perturbation bandwidth on estimated parameters

The parameter identification revealed how reflexive feedback and muscle co-contraction interact to yield the observed admittances. As hypothesized, FTs evoked inhibitory force feedback gains. The corresponding small time delays (25-30 ms) indicate that the force feedback originates from reflexive GTO activity, rather than from slower tactile feedback. The admittance increase of FT with respect to RT was not accompanied by significant changes in intrinsic visco-elasticity or MS feedback, suggesting that the main contributor to the increased admittance was the GTO feedback. As was hypothesized for the RT, little muscle visco-elasticity, MS feedback or GTO feedback were present, indicating that during these perturbations an RT constitutes a good reference condition rather than being well suited for studying reflexes. The small reflexive feedback that was present during RTs was slightly inhibitory for the GTO and slightly excitatory for the MS, which corresponds to the role for the two proprioceptors commonly found in experimental studies that do not consider the impact of functional task instruction.

With regard to the effect of perturbation bandwidth, the parameter identification confirms previous findings (Van der Helm et al. 2002) that at lower perturbation bandwidths, more MS velocity feedback is present than at larger bandwidths. In the present study, the same bandwidth dependency was found for force feedback during FTs: a larger GTO gain was estimated at lower bandwidths. Note that the changes in admittance cannot be explained by changes in muscle visco-elasticity, since no significant changes were found with bandwidth (which was supported by the fact that the mean EMG did not vary with perturbation bandwidth).

Limitation 1: assumption of linearity

The presented method can only be used to describe the linear response to the perturbations. For the current experimental conditions, the linear response generally dominates the observed behavior, as can be concluded from the high coherences and the high VAFs of the linear model fit. However, during FTs the VAFs were lower though the coherences were high. Apparently, the linear part of the behavior could be accurately measured and the model could accurately describe that behavior. However, the lower VAFs indicated that some non-linear behavior was present that could not be described by the model. Close inspection of measured torque and position signals showed that power was present on frequencies where the perturbation did not contain power. Some part of the subject's response did not have a linear relation with the perturbation, which is likely due to intermittent control actions. Most subjects indicated to have more difficulty performing FTs than the other tasks, in particular with the FT with $2.0 \mathrm{~Hz}$ perturbation bandwidth. Note that during FTs, the force can be controlled at different operating points, which allows some drift in the position (reducing the position VAF, but hardly influencing the frequency domain fit).

\section{Limitation 2: parameter interplay}

The human body has redundant mechanisms at its disposal to realize a wide range of neuromuscular dynamics. Consequently, a model that aims to describe the separate contributions of these mechanisms is over-parameterized by definition. The parameter fit procedure utilizes the specific characteristics of each mechanism in the frequency domain. However, due to substantial interplay between these modeled mechanisms, multiple combinations of parameters can yield approximately the same admittance. The parameter fit procedure converged to a unique solution by fitting the model to a diverse set of conditions simultaneously, while assuming certain condition-independent parameters (e.g., inertia, reflexive time delays), which effectively reduced the number of parameters to be fitted.

Confidence in the validity of the parameters was obtained from the good fits in both frequency (Fig. 8) and time domain (Table 4), by good parameter convergence from different starting values and by the fact that the measured mean EMG resembled the estimated visco-elasticity. Moreover, parameter values that have been previously determined (e.g., Kirsch and Kearney 1997) through other means (e.g., inertia, tendon and muscle stiffness, and 
reflexive time delays) converged to similar values as found in the current study.

Limitation 3: spinal versus cortical feedback

Another concern in the choice of the model structure is the possible presence of other feedback pathways: visual and tactile feedback might also be a contributing factor to the low-frequent changes in admittance. However, motion control in response to only visual feedback is possible for up to $6 \mathrm{rad} / \mathrm{s}(\sim 1.0 \mathrm{~Hz})$ at best (McRuer and Jex 1967): the visual control loop is characterized by a relatively large time delay (above $200 \mathrm{~ms}$ ). Although the time delay for the tactile loop is smaller (approximately $120 \mathrm{~ms}$ ), it is still substantially larger than the delays estimated in this study for GTO and MS. Visual and tactile feedback is therefore too slow to explain the significant increase in admittance up to $3.0 \mathrm{~Hz}$ during FTs. Still, there is a potential contribution at low frequencies, and this interplay may lead to reflex gains different from those found in the current study. To investigate this, a brief model study was done where the neuromusculoskeletal model was supplemented with visual and tactile feedback, modeled as feedback gains and large time delays. Parameter estimation of that model resulted in visual and tactile feedback gains explaining some of the low-frequent adaptations. As a result, the reflexive feedback gains were estimated to be somewhat lower, but still the same qualitative trends were observed in the reflexive parameters (GTO sign change from FT to PT and bandwidth effect). Moreover, without reflexive feedback, the model could not accurately describe the observed admittances. In other words, while the quantitative magnitude of the estimated reflexive gains in this study may not be accurate, the qualitative adaptations in GTO and MS feedback are needed to explain the observed behavior.

\section{Limitation 4: short-latency versus long-latency} reflexive feedback

In addition to cortical feedback pathways ("Limitation 3"), an influence of slow spinal feedback can be expected (Grey et al. 2001; Petersen et al. 1998). After all, medium- and long-latency reflexes have been shown to be task dependent (Crago et al. 1976; Rothwell et al. 1980). Implementation of every single physiologically known pathway into the model would certainly result in a more detailed, but also a greatly over-parameterized model (see "Limitation 2"). To investigate whether the main reflexive contribution to the measured behavior was from short or longlatency reflexes, the model parameterization was repeated with a neuromusculoskeletal model where the short-latency reflexes were replaced by long-latency reflexes (time delay fit boundaries of $80-120 \mathrm{~ms}$ ). The solid line in the right panel of Fig. 9 shows the parameter fit with the longlatency reflexes for a typical subject. The parameterization still fitted high feedback gains to yield the large low-frequent admittance changes. However, due to their larger time delays, a trough in the modeled admittance appeared around $1 \mathrm{~Hz}$, which did not correspond to the measured admittance. Note that although it can be concluded from this result that short-latency reflexes are required to accurately fit the data, this does not rule out contributions by medium- and long-latency reflexes.

The fact that short-latency reflexes are required to accurately fit the data in this study could be because it focused on single-joint behavior. It has been suggested in literature that the most important function of the shortlatency reflexes is to compensate for muscle yielding in stretched muscles (Nichols and Houk 1976; Crago et al. 1976; Allum et al. 1982; Allum and Mauritz 1984; Sinkjær et al. 1996). Long-latency reflexes are primarily involved in multi-joint movements (Gielen et al. 1988; Soechting and Lacquaniti 1988; Perreault et al. 2006; Kurtzer et al. 2008), where coordinated muscle activation patterns (such as synergies) are controlled by supraspinal brain regions.

\section{Measuring GTO and MS feedback simultaneously}

The common approach to examine reflexive behavior, based on applying transient perturbations (such as the deep tendon reflex), cannot distinguish between GTO and MS feedback. One way to distinguish contributions of GTO and MS activity is to isolate reflexive activity in response to only muscle stretch, or only muscle force (Doornik and Sinkjær 2008). However, that approach does not apply to motion control tasks where both GTO and MS activity may be present. MS, GTO and muscle visco-elasticity each contribute to the muscle force and mutually affect each other. The current study used closed-loop modeling as a way to distinguish between GTO and MS activity, and as such is the first to identify how GTO and MS feedback act together simultaneously to realize task performance.

\section{Conclusions}

The experiment shows that the dynamic human response to perturbations (i.e., the admittance) is strongly influenced by task instruction and the perturbation bandwidth. Parameter identification of a physiological model provides insight into how feedback from Golgi tendon organs, muscle spindles and muscle co-contraction simultaneously contribute to the observed control behavior.

Relax tasks evoked a large admittance, realized by little co-contraction or reflexive activity. A continuous FT results in an even larger admittance, mainly caused by 
inhibitory Golgi tendon organ feedback, accompanied by little co-contraction. A continuous PT results in a much smaller admittance, mainly caused by high levels of cocontraction and excitatory feedback from Golgi tendon organs, accompanied by excitatory position feedback and inhibitory velocity feedback from muscle spindles. Moreover, during continuous force and position tasks with large bandwidth perturbations, the reflexive feedback is suppressed, most likely to maintain stability.

Although many questions remain about the functionality of Golgi tendon organs, the results indicate that the strength of Golgi tendon organ feedback is adaptable and that it plays an important role during continuous tasks where force or position needs to be controlled.

Acknowledgments We thank E. de Vlugt for support. This research was funded by the Dutch Government Grant BSIK03016.

Open Access This article is distributed under the terms of the Creative Commons Attribution Noncommercial License which permits any noncommercial use, distribution, and reproduction in any medium, provided the original author(s) and source are credited.

\section{Appendix 1: model derivation}

The measured behavior in the main article can be described by a linear motion control model based on a linearized Hill-type muscle model (Van der Helm et al. 2002), of which the linearization is described in Appendix 2.

To assess the influence of the model parameters on the frequency domain and time domain characteristics of the admittance, a graphical user interface was developed previously (Schouten et al. 2008). For clarification, in this study, the equations from which the model is derived are presented below. Note that the model is represented in the frequency domain where $s$ denotes the Laplace operator (see Fig. 2).

The torque that accelerates the total inertia is the sum of the gas pedal torque $T_{c}(s)$ and the opposing torque $T_{\text {muscle }}(s)$ generated by the muscles, according to:

$\theta_{\text {ankle }}(s)=H_{i}(s)\left[T_{c}(s)+T_{\text {muscle }}(s)\right]$

where

$H_{i}(s)=\frac{1}{I_{\mathrm{total}} s^{2}}$

and where $I_{\text {total }}$ represents the total inertia, which consists of the inertia of the lower leg in this configuration, together with the inertia of the setup beyond the force sensor (determined to be $23.3 \mathrm{~g} \mathrm{~m}^{2}$ ).

$T_{\text {muscle }}(s)$ consists of an intrinsic and a reflexive component. The magnitude of these contributions depends on the muscle stretch, which would be linear to the ankle angle for infinite tendon stiffness. This is not a reasonable assumption (especially for the Achilles tendon), so the muscle stretch is modeled as the difference between ankle angle (i.e., total muscle stretch, including the tendon) and tendon stretch, respectively, transformed to $\theta_{\text {ankle }}$ and $\theta_{\text {tendon }}$. The contributions to the muscle torque are described according to:

$T_{\text {muscle }}(s)=H_{\text {act }}(s) A(s)-H_{\text {ve }}(s)\left[\theta_{\text {ankle }}(s)-\theta_{\text {tendon }}(s)\right]$

where $H_{\text {act }}$ describes the activation dynamics (the process of active muscle force buildup following a neural activation signal $A) . H_{\mathrm{ve}}$ describes the intrinsic muscle visco-elasticity according to:

$H_{\mathrm{ve}}(s)=k_{a}+b_{a} s$

with $k_{a}$ the total intrinsic stiffness and $b_{a}$ the total intrinsic damping of (already contracted) muscles. Visco-elasticity can be actively increased by muscle co-contraction, so the two gains are allowed to vary between the conditions.

The tendon angle depends on the tendon stiffness $k_{\text {tendon }}$ and the muscle torque $T_{\text {muscle }}$ according to:

$\theta_{\text {tendon }}(s)=H_{\text {tendon }}(s) T_{\text {muscle }}(s)=k_{\text {tendon }} T_{\text {muscle }}(s)$

$H_{\text {act }}$ is approximated by a second-order system:

$H_{\text {act }}(s)=\frac{\omega_{o}^{2}}{s^{2}+2 d_{a} \omega_{o} s+\omega_{o}^{2}}$

with eigen-frequency $f_{a}$ and relative damping $d_{a}$ and with $\omega_{o}=2 \pi f_{a}$.

The neural activation signal $A(s)$ is modeled as:

$A(s)=U(s)-H_{\text {gto }}(s) T_{\text {muscle }}(s)-H_{\mathrm{ms}}(s)\left[\theta_{\text {ankle }}(s)\right.$

where $U(s)$ represents deviations from the mean supraspinal command. Note that $U(s)$ does not contain the mean descending command that results in co-contraction (which is modeled by intrinsic visco-elasticity $k_{a}$ and $b_{a}$ ). Effectively, $U(s)$ can be assumed to be zero because of the unpredictable nature of the perturbations. $A(s)$ is therefore only the result of feedback from muscle spindles $\left(H_{\mathrm{ms}}\right)$ and Golgi tendon organs $\left(H_{\text {gto }}\right)$.

$H_{\mathrm{ms}}$ models the activity of muscle spindles as a lumped position and velocity feedback with a time delay, according to

$H_{\mathrm{ms}}(s)=\left(k_{p}+k_{v} s\right) e^{-s \tau_{\mathrm{ms}}}$

The parameters $k_{p}$ and $k_{v}$ represent the gains of, respectively, the monosynaptic stretch length and stretch velocity feedback. A single short-latency time delay $\tau_{\mathrm{ms}}$ is used to model the elapsed time due to the neural signal traveling from the spindles to the spinal cord and back to the muscle (De Vlugt et al. 2002). Muscle spindles are often reported to have an excitatory effect on the alpha 
motor neuron: a positive gain results in activity that resists muscle stretch, decreasing the admittance. Negative gains are allowed as well and represent inhibitory effects. GTO dynamics are described by a force feedback gain $k_{f}$ and a time delay $\tau_{\mathrm{gto}}$.

$H_{\mathrm{gto}}(s)=k_{f} e^{-s \tau_{\mathrm{gto}}}$

A positive gain $k_{f}$ denotes an inhibitory effect, increasing the admittance. Since literature has indicated that GTOs can deliver excitatory feedback as well, a negative gain is also allowed in this model.

The pedal rotations not only cause joint rotations, but also displacement of skin and underlying tissues. This effect is modeled as contact dynamics:

$T_{c}(s)=H_{c}(s)\left[\theta_{\text {pedal }}(s)-\theta_{\text {ankle }}(s)\right]$

with $H_{c}$ modeled as:

$H_{c}(s)=b_{c} s+k_{c}$

where $b_{c}$ and $k_{c}$ respectively denote the viscous and elastic part of the contact dynamics. Since in the experiment the foot is strapped to the pedal, these values are expected to be constant over all experimental conditions.

Equations 6-10 are combined into one equation, as can be found in the main text as Equation 3.

\section{Appendix 2: model linearization}

The model is based on previous research on the linearization of a multiplicative Hill-type model (Stroeve 1998), in which the muscle force is described by the multiplication of the activation signal $a$, the force-length relationship $f_{l}$ $\left(L_{m}\right)$, the force-velocity relationship $f_{v}\left(V_{m}\right)$ and the maximal force $F_{\max }$ :

$F_{s}=a \cdot f_{l}\left(L_{m}\right) \cdot f_{v}\left(V_{m}\right) \cdot F_{\max }$

The first-order linearized activation term is a partial derivative of $F_{s}$ to $a$, and is approximated by:

$\partial F_{s} / \partial a=f_{l}\left(L_{m}\right) \cdot f_{v}\left(V_{m}\right) \cdot F_{\max }$

The variations of muscle force $\delta F_{s}$ caused by changing muscle activation $\delta a$ around an operating point can now be described as:

$$
\begin{aligned}
\delta F_{s}= & {\left[f_{l}\left(L_{m}\right) \cdot f_{v}\left(V_{m}\right) \cdot F_{\max }\right] \cdot \delta a } \\
& +\left[a \cdot f_{v}\left(V_{m}\right) \cdot F_{\max } \cdot \partial f_{l}\left(L_{m}\right) / \partial L_{m}\right] \cdot \delta L_{m} \\
& +\left[a \cdot f_{l}\left(L_{m}\right) \cdot F_{\max } \cdot \partial f_{v}\left(V_{m}\right) / \partial V_{m}\right] \cdot \delta V_{m},
\end{aligned}
$$

which can be rewritten in the nomenclature of the current study as:

$$
\begin{aligned}
T_{\text {muscle }}= & {\left[f_{l}\left(L_{m}\right) \cdot f_{v}\left(V_{m}\right) \cdot F_{\max }\right] \cdot \mathrm{A}+\left[k_{a}\right] \theta_{\text {muscle }} } \\
& +\left[b_{a}\right] \dot{\theta}_{\text {muscle }}
\end{aligned}
$$

with $T_{\text {muscle }}$ now denoting deviations in muscle force $\left(\delta F_{s}\right)$, $A$ deviations in muscle activation $(\delta a)$ due to reflexive

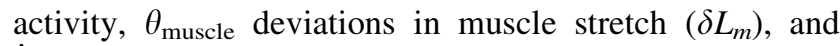
$\dot{\theta}_{\text {muscle }}$ the deviations in muscle stretch velocity $\left(\delta V_{m}\right)$. Note that the intrinsic stiffness and damping due to already contracted muscles is described by $k_{a}$ and $b_{a}$, respectively. The term $\left[f_{l}\left(L_{m}\right), f_{v}\left(V_{m}\right) . F_{\max }\right]$ is for this model implicitly incorporated in the loop gain of each of the reflexive

\begin{tabular}{|c|c|c|}
\hline Parameters & $\begin{array}{l}\text { Lower } \\
\text { boundary }\end{array}$ & $\begin{array}{l}\text { Upper } \\
\text { boundary }\end{array}$ \\
\hline Ankle inertia, $I_{a}\left(\mathrm{~kg} \mathrm{~m}^{2}\right)$ & 0.01 & 0.6 \\
\hline Tendon stiffness, $k_{\text {tendon }}(\mathrm{Nm} / \mathrm{rad})$ & 100 & 10,000 \\
\hline Muscle spindle time delay, $\tau_{\mathrm{ms}}(\mathrm{s})$ & 0.015 & 0.05 \\
\hline $\begin{array}{l}\text { Golgi tendon organ time delay, } \\
\tau_{\text {gto }}(\mathrm{s})\end{array}$ & 0.015 & 0.04 \\
\hline $\begin{array}{l}\text { Relative damping activation } \\
\text { dynamics, } d_{a}(\mathrm{~s})\end{array}$ & 0.5 & 1.5 \\
\hline $\begin{array}{l}\text { Eigen-frequency activation } \\
\text { dynamics, } f_{a}(\mathrm{~Hz})\end{array}$ & 2 & 10 \\
\hline $\begin{array}{l}\text { Contact dynamics viscosity, } b_{c} \\
(\mathrm{Nms} / \mathrm{rad})\end{array}$ & 1 & 100 \\
\hline $\begin{array}{l}\text { Contact dynamics stiffness, } k_{c} \\
(\mathrm{Nm} / \mathrm{rad})\end{array}$ & 100 & 2,000 \\
\hline Muscle viscosity, $b_{a}(\mathrm{Nms} / \mathrm{rad})$ & 0.01 & 20 \\
\hline Muscle stiffness, $k_{a}(\mathrm{Nm} / \mathrm{rad})$ & 100 & 1,500 \\
\hline $\begin{array}{l}\text { Muscle stretch velocity feedback } \\
\text { gain, } k_{v}(\mathrm{Nms} / \mathrm{rad})\end{array}$ & -40 & 40 \\
\hline $\begin{array}{l}\text { Muscle stretch length feedback } \\
\text { gain, } k_{p}(\mathrm{Nm} / \mathrm{rad})\end{array}$ & $-1,000$ & 1,000 \\
\hline Muscle force feedback gain, $k_{f}(-)$ & -10 & 10 \\
\hline
\end{tabular}
feedback loops $\left(k_{p}, k_{v}, k_{f}\right)$.

\section{Appendix 3}

See Tables 2, 3, and 4.

Table 2 Lower and upper parameter boundaries

Table 3 Condition-independent parameters

\begin{tabular}{lc}
\hline Parameters & $\begin{array}{l}\text { Mean } \pm \mathrm{SD} \\
(n=10)\end{array}$ \\
\hline Ankle inertia, $I_{a}\left(\mathrm{~kg} \mathrm{~m}^{2}\right)$ & $0.078 \pm 0.04$ \\
Tendon stiffness, $k_{\text {tendon }}(\mathrm{Nm} / \mathrm{rad})$ & $5,074 \pm 2,369$ \\
Muscle spindle time delay, $\tau_{\mathrm{ms}}(\mathrm{s})$ & $0.038 \pm 0.009$ \\
Golgi tendon organ time delay, $\tau_{\mathrm{gto}}(\mathrm{s})$ & $0.025 \pm 0.009$ \\
Relative damping activation dynamics, $d_{a}(-)$ & $0.99 \pm 0.32$ \\
Eigen-frequency activation dynamics, $f_{a}(\mathrm{~Hz})$ & $2.68 \pm 0.78$ \\
Contact dynamics viscosity, $b_{c}(\mathrm{Nms} / \mathrm{rad})$ & $24.8 \pm 13.6$ \\
Contact dynamics stiffness, $k_{c}(\mathrm{Nm} / \mathrm{rad})$ & $826 \pm 410$ \\
\hline
\end{tabular}


Table 4 Validation of the ARMAX approximations and parameter fits

\begin{tabular}{|c|c|c|c|c|c|}
\hline \multirow[t]{2}{*}{ Task } & \multirow[t]{2}{*}{ Bandwidth (Hz) } & \multicolumn{2}{|l|}{ ARMAX } & \multicolumn{2}{|l|}{ Parameter fit } \\
\hline & & $\mathrm{VAF} T_{c}(\mathrm{SD})(\%)$ & VAF $\theta_{\text {pedal }}(\mathrm{SD})(\%)$ & $\mathrm{VAF} T_{c}(\mathrm{SD})(\%)$ & VAF $\theta_{\text {pedal }}(\mathrm{SD})(\%)$ \\
\hline \multirow[t]{3}{*}{ Force } & 0.7 & $84.3( \pm 5.6)$ & $65.2( \pm 10.9)$ & $39.0( \pm 9.6)$ & $85.1( \pm 7.9)$ \\
\hline & 1.2 & $88.6( \pm 4.8)$ & $50.1( \pm 14.7)$ & $69.7( \pm 2.9)$ & $81.5( \pm 13.1)$ \\
\hline & 2.0 & $91.4( \pm 5.3)$ & $35.6( \pm 12.6)$ & $85.7( \pm 2.0)$ & $73.6( \pm 14.6)$ \\
\hline \multirow[t]{3}{*}{ Relax } & 0.7 & $99.8( \pm 0.1)$ & $99.1( \pm 0.3)$ & $93.3( \pm 1.0)$ & $94.5( \pm 1.9)$ \\
\hline & 1.2 & $99.7( \pm 0.1)$ & $98.8( \pm 0.2)$ & $94.7( \pm 0.9)$ & $96.4( \pm 1.5)$ \\
\hline & 2.0 & $99.7( \pm 0.1)$ & $98.4( \pm 0.3)$ & $94.9( \pm 1.1)$ & $95.4( \pm 1.7)$ \\
\hline \multirow[t]{3}{*}{ Position } & 0.7 & $99.8( \pm 0.1)$ & $78.4( \pm 7.9)$ & $94.9( \pm 0.4)$ & $78.8( \pm 22.3)$ \\
\hline & 1.2 & $99.8( \pm 0.1)$ & $81.9( \pm 6.2)$ & $97.0( \pm 0.2)$ & $84.1( \pm 15.7)$ \\
\hline & 2.0 & $99.8( \pm 0.2)$ & $88.5( \pm 5.6)$ & $97.8( \pm 0.2)$ & $91.2( \pm 8.4)$ \\
\hline
\end{tabular}

Mean and standard deviation of the variance accounted for (VAF) of the ARMAX-approximated signals to the experimental data (left) and model-predicted to the ARMAX-approximated signals (right); calculated for both gas pedal torque $\left(T_{c}\right)$ and gas pedal depression angle $\left(\theta_{\text {pedal }}\right)$ for all subjects

\section{References}

Abbink DA (2006) Neuromuscular analysis of haptic gas pedal feedback during car following. Dissertation, Delft University of Technology, Delft University Press

Abbink DA (2007) Task instruction: the largest influence on human operator control dynamics. In: Proceedings of world haptics, Tsukuba, Japan, 22-24 March 2007, pp 206-211

Allum JHJ, Mauritz KH (1984) Compensation for intrinsic muscle stiffness by short-latency reflexes in human triceps surae muscles. J Neurophys 52:797-818

Allum JHJ, Mauritz KH, Vögele H (1982) The mechanical effectiveness of the short-latency reflexes in the human triceps surae muscles revealed by ischaemia and vibration. Exp Brain Res 48:153-156

Cathers I, O'Dwyer N, Neilson P (1999) Dependence of stretch reflexes on amplitude and bandwidth of stretch in human wrist muscle. Exp Brain Res 129:278-287

Colebatch JG, McCloskey DI (1987) Maintenance of constant arm position or force: reflex and volitional components in man. J Physiol 386:247-261

Crago PE, Houk JC, Hasan Z (1976) Regulatory actions of human stretch reflex. J Neurophysiol 39(5):925-935

De Vlugt E, Van der Helm FCT, Schouten AC (2002) Adaptation of reflexive feedback during arm posture to different environments. Biol Cybern 87:10-26

De Vlugt E, Schouten AC, Van der Helm FCT (2006) Quantification of intrinsic and reflexive properties during multijoint arm posture. J Neurosci Methods 155:328-349

Doemges F, Rack PMH (1992a) Changes in the stretch reflex of the human first dorsal interosseous muscle during different tasks. J Physiol (Lond) 447:563-573

Doemges F, Rack PMH (1992b) Task-dependent changes in the response of human wrist joints to mechanical disturbance. J Physiol (Lond) 447:575-585

Donelan JM, Pearson KG (2004) Contribution of force feedback to ankle extensor activity in decerebrate walking cats. J Neurophysiol 92:2093-2104

Doornik J, Sinkjær T (2008) Robotic platform for human gait analysis. IEEE Trans Biomed Eng 54(9):1696-1702

Faist M, Hoefer C, Hodapp M, Dietz V, Berger W, Duysens J (2006) In humans Ib facilitation depends on locomotion while suppression of $\mathrm{Ib}$ inhibition requires loading. Brain Res 1076:87-92
Geyer H, Seyfarth A, Blickhan R (2003) Positive force feedback in bouncing gaits? Proc Biol Sci 270(1529):2173-2183

Gielen CCAM, Ramaekers L, Van Zuylen EJ (1988) Long-latency stretch reflexes as co-ordinated functional responses in man. J Physiol 407:273-292

Grey MJ, Ladouceur M, Andersen JB, Nielsen JB, Sinkjær T (2001) Group II muscle afferents probably contribute to the medium latency soleus stretch reflex during walking in humans. J Physiol 534(3):925-933

Grey MJ, Nielsen JB, Mazzaro N, Sinkjær T (2007) Positive force feedback in human walking. J Physiol 581:99-105

Hammond PH (1956) The influence of prior instruction to the subject on an apparently involuntary neuro-muscular response. J Physiol 132(1):17-18

Hore J, McCloskey DI, Taylor JL (1990) Task-dependent changes in gain of the reflex response to imperceptible perturbations of joint position in man. J Phyiol 429:309-321

Jaeger R, Gottlieb G, Agarwal G (1982) Myoelectric responses at flexors and extonsors of the human wrist to step torque perturbations. J Neurophysiol 48:388-402

Jankowska E, McCrea DA (1983) Shared reflex pathways from Ib tendon organ afferents and Ia muscle spindle afferents in the cat. J Physiol 338:99-111

Jenkins GM, Watts DG (1968) Spectral analysis and its applications. Holden-Day, San Francisco

Kearney RE, Stein RB, Parameswaran L (1997) Identification of intrinsic and reflex contributions to human ankle stiffness dynamics. IEEE Trans Biomed Eng 44(6):493-504

Kiemel T, Oie K, Jeka JJ (2006) The slow dynamics of postural sway are in the feedback loop. J Neurophysiol 95:1410-1418

Kirsch R, Kearney R (1997) Identification of time-varying stiffness dynamics of the human ankle joint during an imposed movement. Exp Brain Res 114:71-85

Kurtzer IL, Pruszynski JA, Scott SH (2008) Long-latency reflexes of the human arm reflect an internal model of limb dynamics. Curr Biol 18(6):449-453

Lasschuit J, Lam TM, Mulder M, van Paassen MM, Abbink DA (2008) Measuring and modeling neuromuscular system dynamics for haptic interface design. In: Proceedings of the AIAA modeling and simulation technologies conference and exhibit 18-21 August 2008, Honolulu, Hawaii

McRuer DT, Jex HR (1967) A review of quasi-linear pilot models. IEEE Trans Human Factors Electron 8(3):231-249 
Mugge W, Abbink DA, Van der Helm FCT (2007) Reduced power method: how to evoke low-bandwidth behavior while estimating the full-bandwidth dynamics. In: Proceedings of the IEEE 10th international conference on rehabilitation robotics, 13-15 June 2007, pp 575-581

Mugge W, Schuurmans J, Schouten AC, Van der Helm FCT (2009) Sensory weighting of force and position feedback in human motor control tasks. J Neurosci 29(17):5476-5482

Nichols TR, Houk JC (1976) Improvement in linearity and regulation of stiffness that results from actions of stretch reflex. J Neurophysiol 39(1):119-142

Pearson KG, Collins DF (1993) Reversal of the influence of group Ib afferents from plantaris on activity in medial gastrocnemius muscle during locomotor activity. J Neurophysiol 70:1009-1017

Perreault EJ, Chen K, Lewis GN (2006) Regulation of multijoint stretch reflexes during interactions with stiff and compliant environments. In: 28th Annual international conference of the IEEE, Aug 30-Sept 3 2006. Engineering in Medicine and Biology Society, pp 300-302

Petersen N, Christensen LOD, Morita H, Sinkjær T, Nielsen J (1998) Evidence that a transcortical pathway contributes to stretch reflexes in the tibialis anterior muscle in man. $\mathrm{J}$ Physiol 512(1):267-276

Pintelon R, Schoukens J (2001) System Identification: a frequency domain approach. IEEE Press, New York

Pintelon R, Guillaume P, Rolain Y, Schoukens J, van Hamme H (1994) Parametric identification of transfer functions in the frequency domain: a survey. IEEE Trans Autom Contr 39:22432260

Prochazka A, Hulliger M, Zangger P, Appenteng K (1985) 'Fusimotor set': new evidence for alpha-independent control of gammamotoneurones during movement in the awake cat. Brain Res 339(1):136-140

Prochazka A, Gillard D, Bennett DJ (1997) Implications of positive feedback in the control of movement. American Physiological Society 0022-3077/97
Pruszynski JA, Kurtzer I, Scott SH (2008) Rapid motor responses are appropriately tuned to the metrics of a visuospatial task. J Neurophysiol 100:224-238

Rothwell JC, Traub MM, Marsden CD (1980) Influence of voluntary intent on the human long-latency stretch reflex. Nature 286(5772):496-498

Rudomin P, Schmidt RF (1999) Presynaptic inhibition in the vertebrate spinal cord revisited. Exp Brain Res 129:1-37

Schouten AC, Mugge W, Van der Helm FCT (2008) NMClab, a model to assess the contribution of muscle visco-elasticity and afferent feedback to joint dynamics. J Biomech 41(8):1659-1667

Shadmehr R, Krakauer JW (2008) A computational neuroanatomy for motor control. Exp Brain Res 185:359-381

Sinkjær T, Andersen JB, Larsen B (1996) Soleus stretch reflex modulation during gait in humans. J Neurophysiol 76(2):11121120

Soechting JF, Lacquaniti F (1988) Quantitative evaluation of the electromyographic responses to multidirectional load perturbations of the human arm. J Neurophysiol 59(4):1296-1313

Stein RB, Kearney RE (1995) Nonlinear behavior of muscle reflexes at the human ankle joint. J Neurophysiol 73(1):65-72

Stienen AH, Schouten AC, Schuurmans J, van der Helm FCT (2007) Analysis of reflex modulation with a biologically realistic neural network. J Comput Neurosci 23(3):333-348

Stroeve S (1998) Neuromuscular control model of the arm including feedback and feedforward components. Acta Psychol 100(12):117-131

Van den Hof PMJ, Schrama RJP, Bosgra OH, De Callafon RA (1995) Identification of normalized coprime plant factors from closedloop experimental data. Eur J Control 1:62-74

Van der Helm FCT, Schouten AC, De Vlugt E, Brouwn GG (2002) Identification of intrinsic and reflexive components of human arm dynamics during postural control. J Neurosci 119:1-14

Van der Kooij H, van Asseldonk E, van der Helm FCT (2005) Comparison of different methods to identify and quantify balance control. J Neurosci Methods 145(1-2):175-203 